\title{
Radial displacement of clinical target volume in node negative head and neck cancer
}

\author{
Wan Jeon, MD', Hong-Gyun Wu, MD ${ }^{1,2,3}$, Sang Hyuk Song, MD', Jung-In Kim, MS ${ }^{4,5}$ \\ ${ }^{1}$ Department of Radiation Oncology, ${ }^{2}$ Cancer Research Institute, Seoul National University College of Medicine, Seoul; \\ ${ }^{3}$ Institute of Radiation Medicine, Medical Research Center, Seoul National University, Seoul; ${ }^{4}$ Department of Radiation \\ Oncology, Kangbuk Samsung Hospital, Seoul; ${ }^{5}$ Department of Radiation Oncology, Interdisciplinary Program in Radiation \\ Applied Life Science, Seoul National University College of Medicine, Seoul, Korea
}

Purpose: To evaluate the radial displacement of clinical target volume in the patients with node negative head and neck (HEtN) cancer and to quantify the relative positional changes compared to that of normal healthy volunteers.

Materials and Methods: Three node-negative HEIN cancer patients and five healthy volunteers were enrolled in this study. For setup accuracy, neck thermoplastic masks and laser alignment were used in each of the acquired computed tomography (CT) images. Both groups had total three sequential CT images in every two weeks. The lymph node (LN) level of the neck was delineated based on the Radiation Therapy Oncology Group (RTOG) consensus guideline by one physician. We use the second cervical vertebra body as a reference point to match each $\mathrm{CT}$ image set. Each of the sequential $\mathrm{CT}$ images and delineated neck LN levels were fused with the primary image, then maximal radial displacement was measured at $1.5 \mathrm{~cm}$ intervals from skull base (SB) to caudal margin of LN level $V$, and the volume differences at each node level were quantified.

Results: The mean radial displacements were $2.26( \pm 1.03) \mathrm{mm}$ in the control group and $3.05( \pm 1.97)$ in the HetN cancer patients. There was a statistically significant difference between the groups in terms of the mean radial displacement $(p=0.03)$. In addition, the mean radial displacement increased with the distance from SB. As for the mean volume differences, there was no statistical significance between the two groups.

Conclusion: This study suggests that a more generous radial margin should be applied to the lower part of the neck LN for better clinical target coverage and dose delivery.

Keywords: Head and neck neoplasms, Radiotherapy, Image-guided, Lymph nodes

\section{Introduction}

The head and neck (HEtN) lymph nodes region consists of approximately 300 lymph nodes, which are the most frequent sites of HEtN cancer recurrence and metastasis [14]. Each lymph node (LN) level has anatomical boundaries. For computed tomography (CT) based delineation of clinical target volume in node negative HEtN cancer patients, representatives of major cooperative groups in Europe (Danish Head and Neck Cancer Group, DAHANCA; European Organization for Research and Treatment of Cancer, EORTC; Groupe d'Oncologie Radiotherapie Tete Et Cou, GORTEC) and in North America (National Cancer Institute of Canada, NCIC; Radiation Therapy Oncology Group, RTOG) published consensus guidelines

Received 2 March 2012, Revised 20 March 2012, Accepted 22 March 2012.

Correspondence: Hong-Gyun Wu, MD, Department of Radiation Oncology, Seoul National University College of Medicine, 103 Daehak-ro, Jongno-gu, Seoul 110-799, Korea. Tel: +82-2-2072-3177, Fax: +82-2-765-3317, E-mail: wuhg@snu.ac.kr

(c) This is an Open Access article distributed under the terms of the Creative Commons Attribution Non-Commercial License (http://creativecommons.org/ licenses/by-nc/3.0/) which permits unrestricted non-commercial use, distribution, and reproduction in any medium, provided the original work is properly cited. www.e-roj.org 
in 2003 [5]. This guideline standardized the procedure of contouring the each LN level in node-negative H\&tN cancer patients.

The anatomy of H\&N regions are easily affected by slight movement, due to their complexity in anatomical structure. For radiotherapy, especially in the era of intensity-modulated radiotherapy (IMRT), small intra and inter-fractional anatomical changes could ultimately lead to an inappropriate dose distribution of the target volume [6-8]. Overcoming these variations in HEtN RT, various immobilization devices and different patient setup methods, using electronic portal image or cone beam CT were developed and clinically applied [9-13].

Many patients receiving fractionated RT for H\&N cancer, however, have marked anatomic changes during their course of treatment due to the shrinkage of their primary tumor or nodal mass, resolving post-operative changes, edema, overall body habitus, and weight loss [14]. No consensus guidelines for node negative (NO) neck delineation include a safety margin for organ motion and setup uncertainty, so the decision on the planning target volume (PTV) is usually up to an individual radiation oncologist or experience of each center.

In the era of image-guided radiotherapy (IGRT), online autonomic image analysis and real-time setup correction are utilized in many centers [15]. Although these procedures consider the treatment region, however, the setup correction methods are routinely based on the bony anatomy. Due to its' easy applicability, bony anatomy as a reference point in patient setup does not consider the change of the related soft tissue. It could inevitably lead uncertainties in the coverage of the target volume and make difficulties in calculation of the actual delivered radiation dose.

In this study, using three sequential CT images with neck immobilization device, we evaluate the radial displacement of each neck node level as a surrogate value in inter-fractional change during radiotherapy. In addition, we also evaluate the changes of each neck node level volume from sequential CT images. And to determine the reference radial displacement and No neck node level volume, we enrolled five normal healthy volunteers as a control group.

\section{Materials and Methods}

Five patients who had newly diagnosed node-negative HEtN cancer, were treated with definitive radiotherapy and concurrent chemotherapy. All the patients had pathologically proven diseases, and their regional lymph nodes were evaluated through a physical examination, $\mathrm{CT}$, and whole body positron emission tomography (WB-PET). All the patients were treated with dynamic multileaf collimator (MLC) IMRT. Total radiation dose to gross tumor and fraction size was 67.5 Gy and $2.25 \mathrm{~Gy}$, respectively. The normal control group consists of five volunteers who had no previous history of HEN abnormalities, including benign diseases. All the volunteers and patients signed the study-specific informed consent forms. For acquisition of each CT images, using minimum field of view and radiation exposure less than $1.6 \mathrm{mSv}$ per CT image, we observe the National Council on Radiation Protection and Measurements (NCRP) public dose limit recommendation for ionizing radiation [16].

\section{Image registration}

Three sets of images were acquired from both groups using a CT simulator (Brilliance CT Big Bore; Philips, Andover, MA, USA) and neck immobilization device (Aquaplast thermoplastic, Opti-Mold; WFR Aquaplast Corp., Wickoff, NJ, USA). CT image registration was done in two-week intervals. The first sets were taken with radio-contrast-enhanced CT images, and the rest, in the form of non-enhanced CT images. The normal control group underwent CT scans three times with two-week interval. The first contrast-enhanced CT scans of the patient groups were taken from the planning $\mathrm{CT}$ images, and the second and third non-enhanced CT images were taken at the second and fourth week of treatment, respectively. We used CT scan with a 3-mm slice thickness through the skull base to upper sternal margin of clavicle.

\section{Cervical lymph node contouring method}

All the acquired CT images were imported into the treatment planning system (TPS, Eclipse 8.6 version; Varian Medical Systems, Palo Alto, CA, USA). According to the consensus guidelines on NO neck delineation, all $L N$ levels were manually contoured on all the axial images of both groups (Fig. 1). As a bony landmark for image fusion, the second cervical vertebra, axis was also manually contoured on each CT scan (Fig. 2). For minimizing inter-observer variations in our data, delineation of all LN levels in each acquired CT scan was contoured by the same physician. Moreover, after all the LN levels were delineated, two radiation oncologists at our department reviewed the neck $L N$ level delineation. 

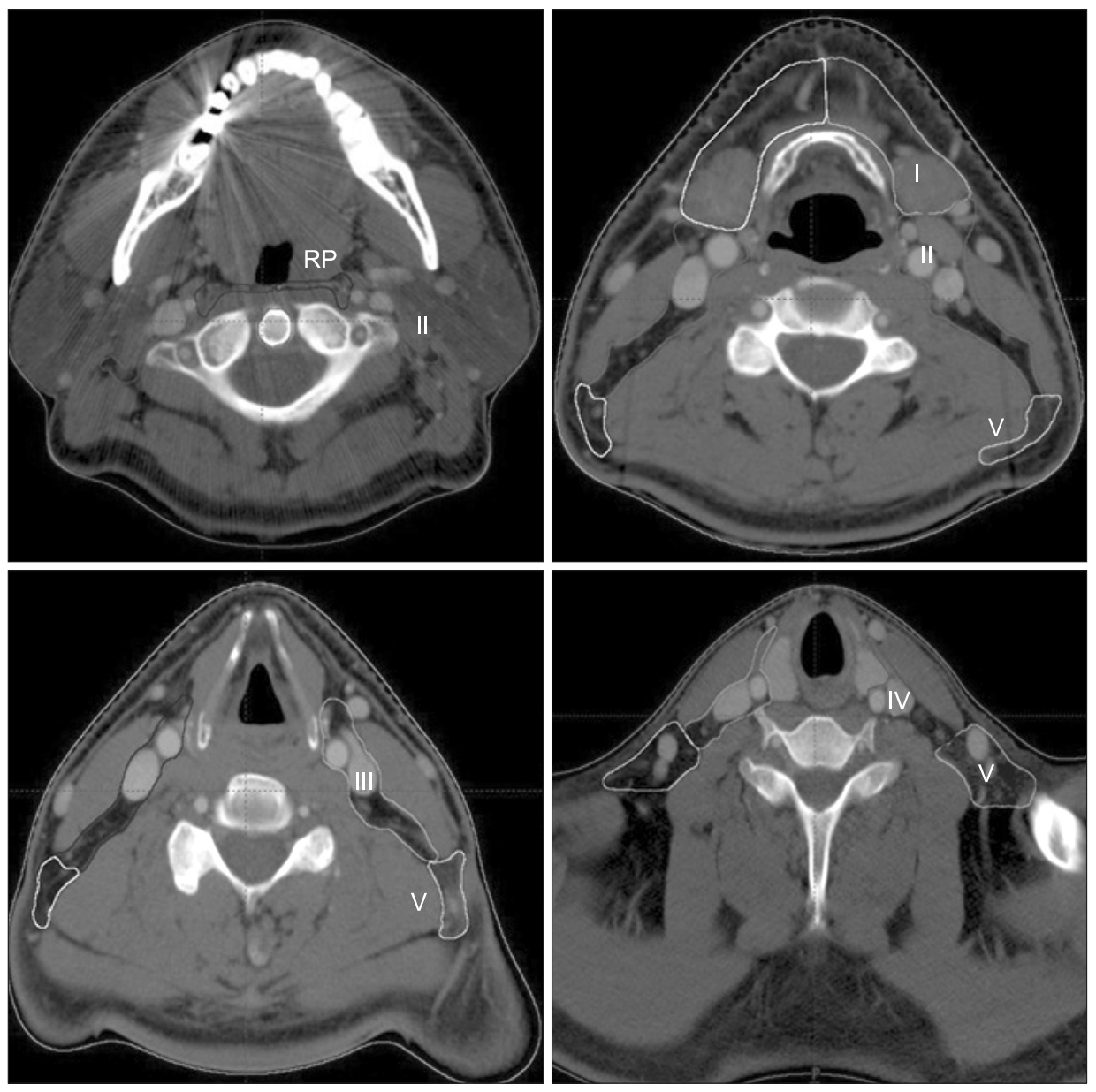

Fig. 1. Delineation of each neck node according to Radiation Therapy Oncology Group (RTOG) consensus guideline.
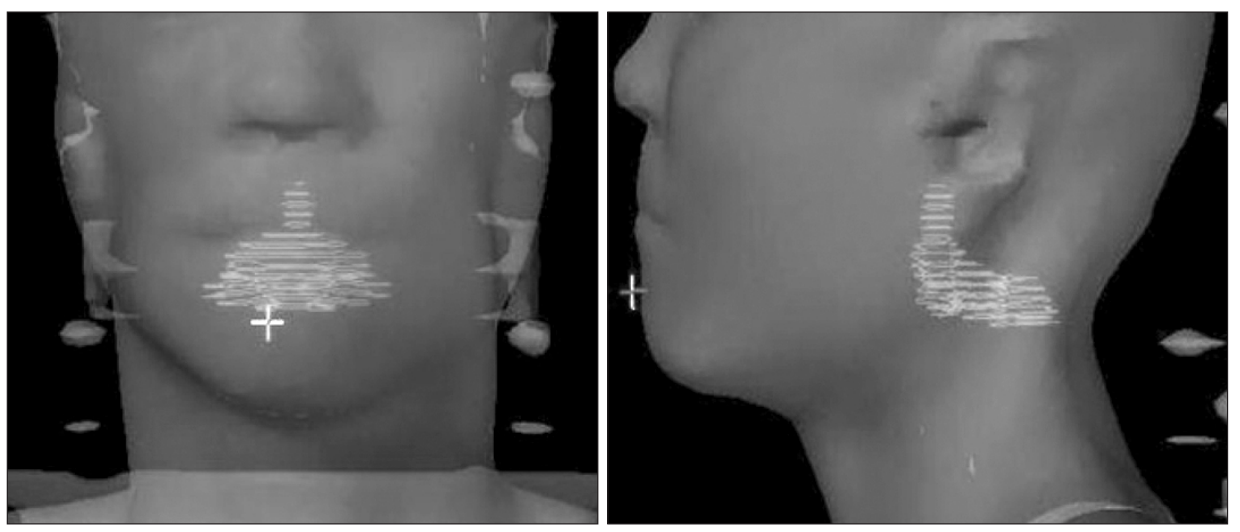

Fig. 2. Cervical vertebra delineation (C2, axis) for bony landmark based fusion.

\section{Evaluation of the radial displacement and volume differences}

Each sequential CT images and delineated neck LN levels were fused with the primary image through TPS rigid registration algorithm, which is automatic image fusion system with certain point. Our reference point for the image fusion was the C2 vertebra. After image fusion, the radial displacement was quantified manually, where the maximum delineation discrepancy exist. The radial displacement evaluated in each $1.5-\mathrm{cm}$ interval from skull base (SB) to the inferior margin of level $V$ (Fig. 3). To eliminate intra and inter observer error in the manual measurement of the radial displacement, it was also reviewed by two radiation oncologists. The mean radial displacement was calculated that dividing the sum of maximal 
displacements between the primary and serial CT scans at each interval by two (total number of CT image acquisition 1). The group mean radial displacement was calculated that dividing the sum of mean displacements in the same group by the total number of each group. To quantify the volume of each LN level from the registered axial images, TPS volume interpolation algorithm, which is automatic volume calculation system for delineated image was used in each registered CT images. From the primary and serial CT images in each group, the mean volume differences were calculated that dividing the sum of the volume differences at each LN level by two (total number of CT image acquisition - 1). The group mean volume difference was calculated that dividing the sum of mean volume differences in each group by the total number of each group.

\section{Statistical analysis}

Mann-Whitney tests was used to compare the quantitative and ordered variables, and Spearman's rho correlation coefficient was used to analyze the correlation between the LN levels and the displacement or volume changes in LN in each group. The study protocol was reviewed and then approved
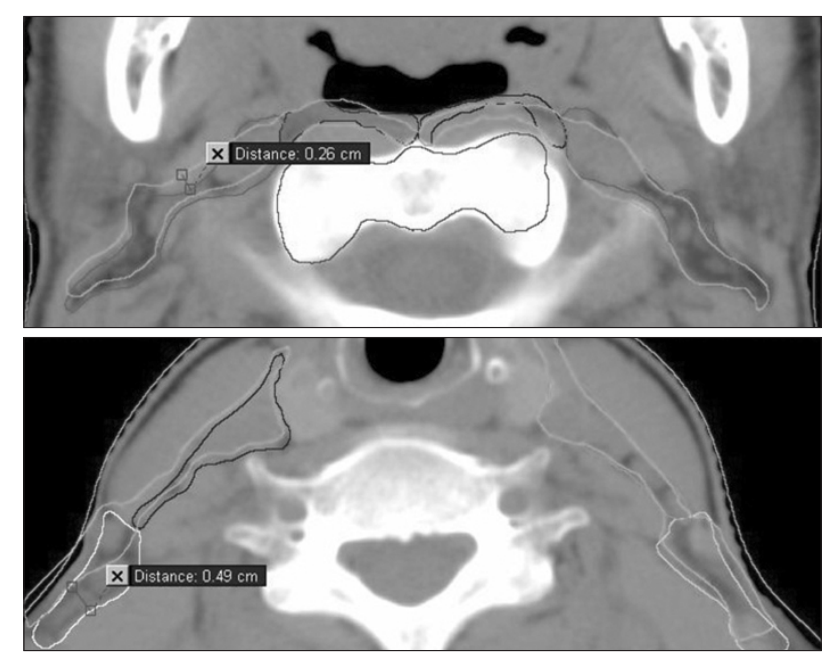

Fig. 3. Manual measurement of maximal radial displacement at 1.5 $\mathrm{cm}$ interval after bony landmark (C2, axis) based fusion. by the institutional review board. The recommendations of the Declaration of Helsinki for biomedical research involving human subjects were also reflected.

\section{Results}

From June to September 2010, five node-negative H\&N cancer patients and five normal volunteers were enrolled in this study. After first CT acquisition, however, two patients declined further study. As a result, total three patients were enrolled and each patient characteristics are shown in Table 1. The five volunteers and three patients successfully underwent serial CT scans. All the patients underwent concurrent chemoradiotherapy and IMRT during study period.

The tendency of radial displacement increased with the distance from SB to LN level V inferior margin (Fig. 4A). The maximal radial displacement occurred at $10.5 \mathrm{~cm}$ from SB in both groups. Each group's mean radial displacement is shown in Table 2. The correlation between the distance from SB and radial displacement was evaluated with Spearman's rho correlation coefficient. The correlation coefficient was 0.638 and statistically significant $(p<0.001)$ (Fig. 4B). The patient group had a greater radial displacement than control group in each measured point. In addition, mean radial displacement was also evaluated according to each group and the values at $6-\mathrm{cm}$ and $10.5-\mathrm{cm}$ distances from SB showed statistical significance ( $p=0.011$ and $p=0.005$, respectively). As for the total mean displacement, the patient group had a greater radial displacement $(3.05 \pm 1.97 \mathrm{~cm})$ than the normal control group $(2.26 \pm 1.03 \mathrm{~cm})$, and their difference was also showed statistical significance $(p=0.03)$.

For the volume differences in each group, no correlation between the mean volume and each LN level was observed. (Fig. 5A, B). The mean volume differences of each group were calculated at each neck node level and they showed no statistical significance (Table 3). About for the total mean volume differences, there was also no statistical significance in each group.

Table 1. Patient characteristics

\begin{tabular}{cccccc}
\hline No. & Age/gender & Primary site & TNM stage & CCRT & RT technique \\
\hline 1 & $61 / \mathrm{F}$ & Buccal mucosa & cT4N0 & Yes & IMRT \\
2 & $48 / \mathrm{F}$ & Nasopharynx & cT4N0 & Yes & IMRT \\
3 & $53 / \mathrm{M}$ & Nasopharynx & cT1N0 & Yes & IMRT \\
\hline
\end{tabular}

TNM, tumor, nodes, metastasis; CCRT, concurrent chemo-radiotherapy; IMRT, intensity-modulated radiation therapy. 
A

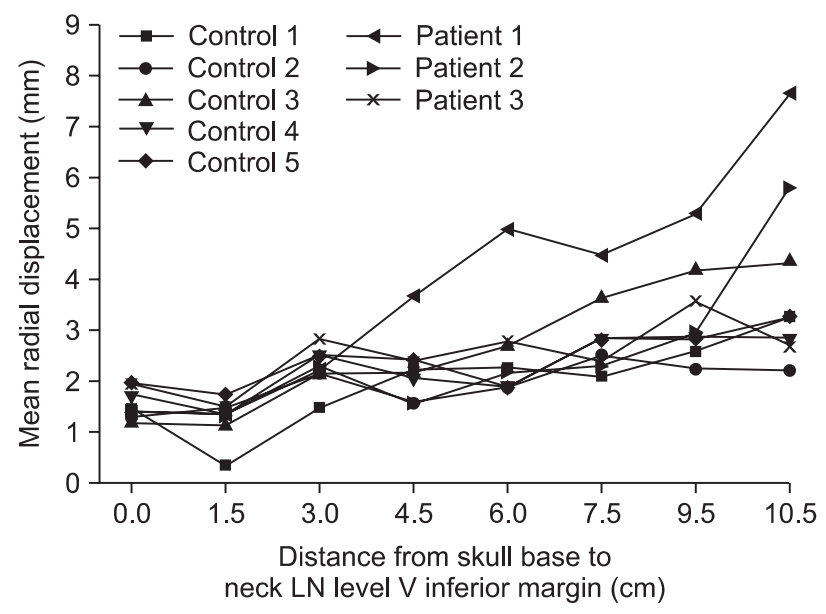

B

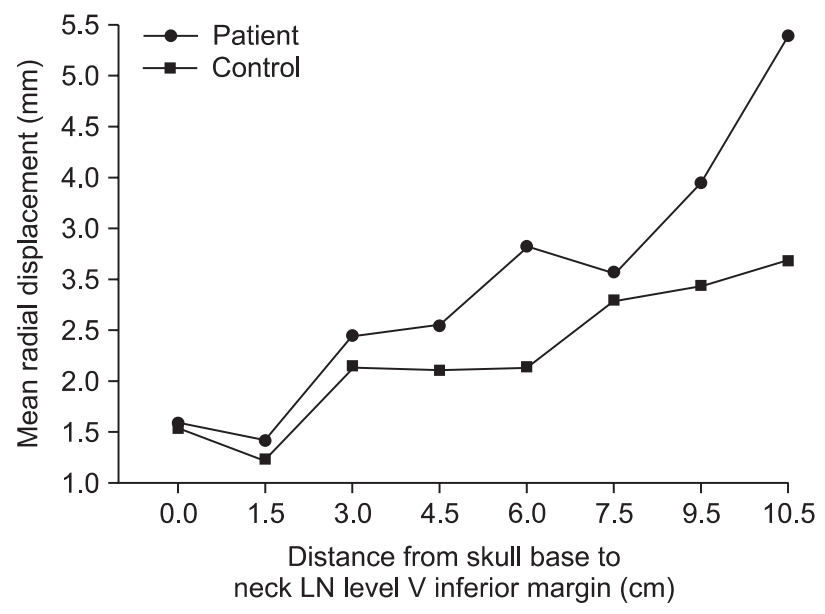

Fig. 4. (A) Mean radial displacement in patients and control group (from skull base to neck lymph node (LN) level V inferior margin at 1.5 $\mathrm{cm}$ interval), (B) group mean radial displacement from skull base to level $V_{;}$Spearman's rho correlation coefficient $=0.638, p<0.001$.

Table 2. Mean radial displacement from skull base to inferior margin of level $V$ lymph node in each group $(\mathrm{mm})$

\begin{tabular}{lccc}
\hline & Control $(n=5)$ & Patient $(n=3)$ & p-value $^{\text {a) }}$ \\
\hline Skull base & $1.54 \pm 0.64$ & $1.51 \pm 0.40$ & 0.846 \\
$1.5 \mathrm{~cm}$ & $1.16 \pm 0.71$ & $1.47 \pm 0.48$ & 0.746 \\
$3 \mathrm{~cm}$ & $2.16 \pm 0.89$ & $2.33 \pm 0.61$ & 0.779 \\
$4.5 \mathrm{~cm}$ & $2.11 \pm 0.41$ & $2.61 \pm 1.24$ & 0.475 \\
$6 \mathrm{~cm}$ & $2.13 \pm 0.57$ & $3.31 \pm 1.53$ & 0.011 \\
$7.5 \mathrm{~cm}$ & $2.81 \pm 0.85$ & $3.13 \pm 1.19$ & 0.588 \\
$9 \mathrm{~cm}$ & $2.92 \pm 0.96$ & $3.96 \pm 1.72$ & 0.067 \\
$10.5 \mathrm{~cm}$ & $3.22 \pm 1.30$ & $5.62 \pm 2.80$ & 0.005 \\
Total & $2.26 \pm 1.03$ & $3.05 \pm 1.97$ & 0.030 \\
\hline
\end{tabular}

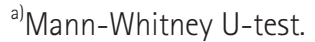

\section{Discussion and Conclusion}

In this study, the anatomic and soft tissue changes were observed in the patients with NO neck disease. These changes were observed over time, despite the autonomic and manual correction based on bony anatomy. Even in the normal control group, radial displacement larger than $3 \mathrm{~mm}$ was observed. This implies that most current practices using bony landmarks as for offline and online setup correction need more attention to encompass these displacements. As for IMRT, with highly conformal treatment technique, steep dose gradients between a tumor and the avoidance structure could be established. Therefore, safety margin around the clinical target volume is a crucial for adequate tumor control. Current CT-based delineation of the LN levels and the relative clinical target volume (CTV) in the node-negative neck (i.e., the DAHANCA, EORTC, GORTEC, NCIC, and RTOG consensus guidelines) mentioned that implementation of these guidelines in the daily practice could contribute to reduced treatment variations from patient to patient and help to conduct multi-institutional clinical trials and/or retrospective studies. In this perspective, several previous reports introduced various kinds of correction methods for set-up errors (vector range, 2 to $7 \mathrm{~mm}$ ) [913]. But these reports did not focus on soft tissue changes, which occurred during treatment time. Using automatic bony landmark (C2, axis) based fusion in TPS, we could only focused on radial displacement of target volume. We performed serial CT scans in both the patient and normal group, and evaluated the radial displacement from SB to lower margin of neck $L N$ level $V$ and the volumes of each neck $L N$ level. The radial displacement in the normal control group as a reference value showed about $3 \mathrm{~mm}$. It was less than that in the patient group, but should be considered as a minimum reference value of displacement. For volume changes of each neck LN levels, there were no statistically significant volume changes in both group.

Our study had several limitations in the number of study population, physician dependant contouring method, manual measurement of the radial displacement and the use of second cervical vertebra as a bony landmark for the CT image fusion.

At first, we enrolled 5 patients and 5 normal volunteers as control group in this study. Of these study population, two patients who did not want to take second CT images, were dropped from this study. Due to small number of node 
A

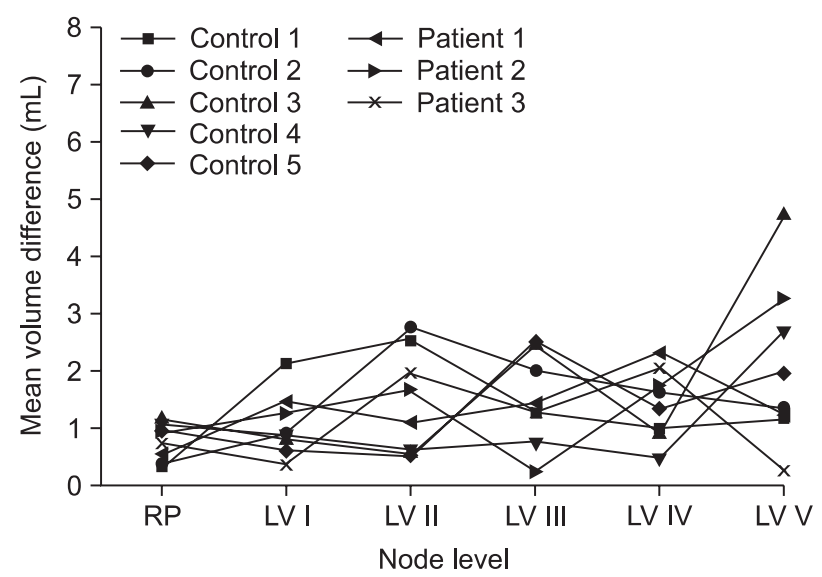

B

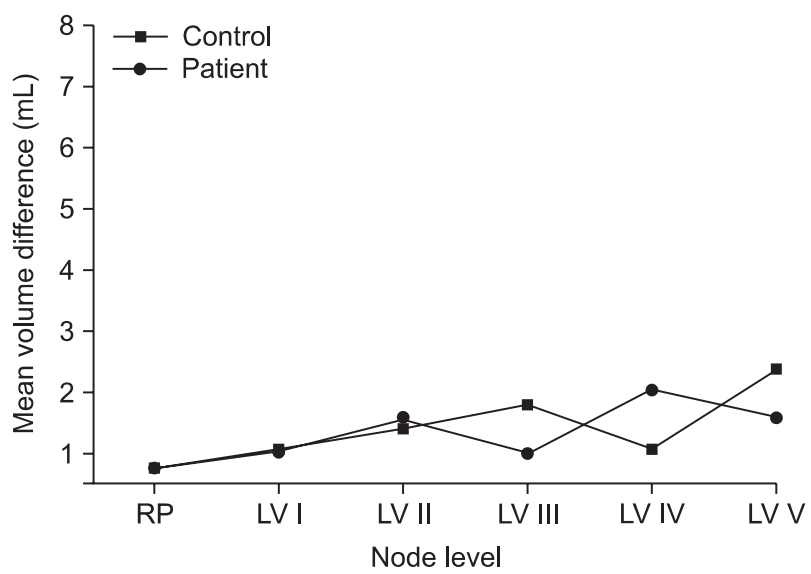

Fig. 5. (A) Mean volume differences at each node level, (B) group mean volume differences at each node level. RP, retropharyngeal; LV, level.

Table 3. Mean volume differences at each node level in each group $(\mathrm{mL})$

\begin{tabular}{lccc}
\hline & Control $(n=5)$ & Patient $(n=3)$ & $p$-value \\
\hline Retropharyngeal & $0.78 \pm 0.42$ & $0.76 \pm 0.39$ & 0.925 \\
Level I & $1.08 \pm 0.64$ & $1.04 \pm 0.89$ & 0.448 \\
Level II & $1.42 \pm 1.14$ & $1.59 \pm 0.50$ & 0.448 \\
Level III & $1.80 \pm 0.99$ & $1.00 \pm 0.68$ & 0.083 \\
Level IV & $1.07 \pm 0.81$ & $2.05 \pm 1.03$ & 0.065 \\
Level V & $2.39 \pm 1.44$ & $1.61 \pm 1.49$ & 0.193 \\
Total & $1.42 \pm 1.07$ & $1.34 \pm 0.95$ & 0.874 \\
\hline
\end{tabular}

a) Mann-Whitney U-test.

negative HCtN cancer patients and low patient accrual rate, we continued our study only with 3 patients. Thus, evaluation of radial displacement and LN level volume changes in patients group had inevitably less clinical implication, despite of statistical significance in radial displacement. Secondly, physician dependant contouring method and manual measurement of the radial displacement might have inter-and intra-observer variations in spite of peer review. Finally, using the second cervical vertebra as the bony landmark for image fusion, the feasibility of this landmark should be evaluated. van Kranen et al. [12], reported that if all the movements were referenced to upper cervical vertebrae (C1-3), there would be almost no residual setup error; but it could be increased in proportion to the distance from the cranial to the caudal direction. Our study also showed the same tendency with radial displacement in both group.

Through this study, we could observe that lower neck LN level showed more radial displacement than upper neck LN level.
Current use of offline and online setup correction methods, using bony anatomy is not sufficient to compensate those uncertainties. Generally, a clinically applied safety margin of $5 \mathrm{~mm}$ includes the delineation error and the radial or craniocaudal setup error. This study showed that as a reference value, the normal control group had an about $3 \mathrm{~mm}$ radial displacement despite of bony landmark based image fusion and the patient group had a more radial displacement in all neck LN levels. Weight loss and the shrinkage of primary tumor during the treatment period might be the cause of these differences.

In conclusion, to overcome these uncertainties caused by neck node delineation, an adequate internal margin, more than $3 \mathrm{~mm}$ might be needed to cover the clinical target volume in the patients with node negative H\&N cancer. For better tumor control and dose delivery to regional lymphatics at risk, more generous radial margin should be considered to the lower neck LN level.

\section{Conflict of Interest}

No potential conflict of interest relevant to this article was reported.

\section{Acknowledgments}

This study was supported by a grant of the Korean Health Technology RetD Project, Ministry of Health \& Welfare, Republic of Korea (A111098). 


\section{References}

1. Ferlito A, Rinaldo A, Devaney KO, et al. Prognostic significance of microscopic and macroscopic extracapsular spread from metastatic tumor in the cervical lymph nodes. Oral Oncol 2002;38:747-51.

2. Magnano M, De Stefani A, Lerda W, et al. Prognostic factors of cervical lymph node metastasis in head and neck squamous cell carcinoma. Tumori 1997;83:922-6.

3. Mamelle G, Pampurik J, Luboinski B, Lancar R, Lusinchi A, Bosq J. Lymph node prognostic factors in head and neck squamous cell carcinomas. Am J Surg 1994;168:494-8.

4. Stringer SP. Current concepts in surgical management of neck metastases from head and neck cancer. Oncology (Williston Park) 1995;9:547-54.

5. Gregoire $V$, Levendag P, Ang KK, et al. CT-based delineation of lymph node levels and related CTVs in the node-negative neck: DAHANCA, EORTC, GORTEC, NCIC, RTOG consensus guidelines. Radiother Oncol 2003;69:227-36.

6. van Asselen B, Raaijmakers CP, Lagendijk JJ, Terhaard CH. Intrafraction motions of the larynx during radiotherapy. Int J Radiat Oncol Biol Phys 2003;56:384-90.

7. Linthout N, Verellen D, Tournel K, Storme G. Six dimensional analysis with daily stereoscopic $x$-ray imaging of intrafraction patient motion in head and neck treatments using five points fixation masks. Med Phys 2006;33:504-13.

8. Zhang L, Garden AS, Lo J, et al. Multiple regions-of-interest analysis of setup uncertainties for head-and-neck cancer radiotherapy. Int J Radiat Oncol Biol Phys 2006;64:1559-69.

9. Gilbeau L, Octave-Prignot $M$, Loncol T, Renard L, Scalliet $P$, Gregoire V. Comparison of setup accuracy of three different thermoplastic masks for the treatment of brain and head and neck tumors. Radiother Oncol 2001;58:155-62.

10. de Boer HC, van Sornsen de Koste JR, Creutzberg CL, Visser $A G$, Levendag PC, Heijmen BJ. Electronic portal image assisted reduction of systematic set-up errors in head and neck irradiation. Radiother Oncol 2001;61:299-308.

11. Boda-Heggemann J, Walter C, Rahn A, et al. Repositioning accuracy of two different mask systems-3D revisited: comparison using true 3D/3D matching with cone-beam CT. Int J Radiat Oncol Biol Phys 2006;66:1568-75.

12. van Kranen $S$, van Beek $S$, Rasch $C$, van Herk M, Sonke JJ. Setup uncertainties of anatomical sub-regions in head-andneck cancer patients after offline CBCT guidance. Int J Radiat Oncol Biol Phys 2009;73:1566-73.

13. Pehlivan $B$, Pichenot $C$, Castaing $M$, et al. Interfractional setup errors evaluation by daily electronic portal imaging of IMRT in head and neck cancer patients. Acta Oncol 2009;48:440-5.

14. Barker JL Jr, Garden AS, Ang KK, et al. Quantification of volumetric and geometric changes occurring during fractionated radiotherapy for head-and-neck cancer using an integrated CT/linear accelerator system. Int J Radiat Oncol Biol Phys 2004;59:960-70.

15. McBain CA, Henry AM, Sykes J, et al. X-ray volumetric imaging in image-guided radiotherapy: the new standard in ontreatment imaging. Int J Radiat Oncol Biol Phys 2006;64:62534.

16. National Council on Radiation Protection and Measurements. Recent application of the NCRP public dose limit recommendation for ionizing radiation. Bethesda: National Council on Radiation Protection and Measurements; 2004. NCRP statement no. 10. 